\title{
Loss of pyrethroid resistance in newly established laboratory colonies of Aedes aegypti.
}

Farah Z. Vera-Maloof ${ }^{a}$, Karla Saavedra-Rodriguez ${ }^{\mathrm{a}}$, Rosa P. Penilla-Navarro ${ }^{\mathrm{b}}$, Americo D. Rodriguez-

Ramirez ${ }^{\mathrm{b}}$, Felipe Dzulc, Pablo Manrique ${ }^{\mathrm{d}}$, William C. Black IV ${ }^{\mathrm{a}^{*}}$.

${ }^{a}$ Colorado State University, College of Veterinary Medicine and Biomedical Sciences

Department of Microbiology, Immunology and Pathology, Arthropod Borne and Infectious Diseases

Laboratory, 1692 Campus Delivery, Fort Collins, Colorado 80523-1692

${ }^{\mathrm{b}}$ Centro Regional de Investigación en Salud Pública, Instituto Nacional de Salud Pública, Colonia Centro, Tapachula, Chiapas 30700, México.

${ }^{\mathrm{c}}$ Centro Nacional de Programas Preventivos y Control de Enfermedades, Benjamín Franklin

132, Escandón, Ciudad de México 11800, México.

d Universidad Autonoma de Yucatan, México, Calle 60 491A, Parque Santa Lucia, Centro, Mérida, Yucatan 97000, Mexico.

* Corresponding author wcb4@colostate.edu

\section{Short title: Loss of pyrethroid resistance}

Keywords: Aedes aegypti, pyrethroid, loss of resistance 


\section{Abstract}

\section{Background}

Resistance to pyrethroid insecticides in Aedes aegypti has become widespread after almost two decades of their frequent use to reduce arbovirus transmission. Despite this, use of pyrethroids continues because they are relatively inexpensive and because of their low human toxicity. Resistance management has been proposed as a means to retain the use of pyrethroids in natural populations. A key component of resistance management assumes that there is a negative fitness associated with resistance alleles so that when insecticides are removed, resistance alleles will decline in frequency. At least three studies in $A e$. aegypti have demonstrated a decrease in pyrethroid resistance once the insecticide is removed.

\section{Methods/Principal Findings}

The present study aims to evaluate variation in the loss of pyrethroid resistance among newly established laboratory populations of Ae. aegypti from Mexico. Eight field collections were maintained for up to eight generations and we recorded changes in the frequencies of mutations at the V1,016I locus and at the $\mathrm{F} 1,534 \mathrm{C}$ locus in the voltage gated sodium channel (VGSC) gene. I1,016 and C1,534 confer resistance. We also examined resistance ratios (RR) with type 1 and 2 pyrethroids.

\section{Conclusions/Significance}

We demonstrate that, in general, the frequency of the Ae. aegypti pyrethroid resistance alleles I1,016 and $\mathrm{C} 1,534$ decline when they are freed from pyrethroid pressure in the laboratory. However, the pattern of decline is strain dependent. In agreement with earlier studies, RR was positively correlated with I1,016 resistant allele frequencies and showed significant protection against permethrin, and deltamethrin whereas F1534C showed protection against permethrin but not against deltamethrin. 


\section{Author Summary}

The author is interested in the evolution of genes that confer resistance to insecticides, especially when this evolution affects binding of insecticides to their target site. The Voltage Gated Sodium Channel gene represents an excellent opportunity to understand how mutations at the target site(s) affect the evolution of resistance in many different pest insect species including Aedes aegypti, the primary vector of Dengue Virus, Yellow Fever, Zika and Chikungunya arboviruses. 


\section{Introduction}

After almost two decades of frequent pyrethroid use for Aedes aegypti (L.) control there is now widespread resistance $[1,2]$. Despite this, use of pyrethroids continues because they are relatively inexpensive and because they have low human toxicity. Resistance management has been proposed as a means of retaining the use of pyrethroids in natural populations [2]. A key component of resistance management assumes that there will be a negative fitness associated with resistance alleles so that when insecticides are removed, resistance alleles will decline in frequency.

Laboratory strains of Ae. aegypti have shown a decrease in resistance once pyrethroid is removed suggesting a fitness cost associated with resistance. To date three laboratory studies have evaluated the loss of pyrethroid resistance in Ae. aegypti. In Taiwan, a permethrin resistant laboratory strain was maintained for 47 generations under permethrin pressure. Following 15 generations without exposure there was a significant decrease in permethrin resistance ratio (RR) and resistance alleles in the Voltage Gated Sodium Channel (VGSC) gene [3]. In Brazil, after 15 generations, the frequency of I1,016 decreased from 0.75 to 0.20 [4]. A study in Mexico, showed a significant increase in the proportion of knocked-down mosquitoes after 10 generations following removal of pyrethroids [5] but without a decrease in the frequency of I,1016 and C1,534 mutations in the VGSC. All three studies were done in laboratory cages.

The present study aims to evaluate the loss of pyrethroid resistance from eight collections of $A e$. aegypti, (six field collections from or near the city of Merida and two collections from Tapachula and Acapulco from southern Mexico). These collections were maintained without pyrethroid pressure for eight consecutive generations during which we recorded changes in the frequencies of the two VGSC mutations I1,016 and C1,534, and the analysis of resistance ratios (RR) with permethrin (pyrethroid type 1) and deltamethrin (pyrethroid type 2). 


\section{Materials and Methods}

\section{Aedes aegypti field populations}

In 2014, larvae were collected from eight public sites in Mexico. These were reared to adults and identified as Ae. aegypti. These were then blood fed (citrated sheep blood - Colorado Serum Co., Denver Colorado) in an artificial membrane feeder and eggs were collected for shipment. The GPS coordinates and name abbreviations of the collection sites appear in Table 1. Eggs from Yucatan were collected from three sites in urban areas of Merida and three collections from villages near Merida. Two additional collections were made in Tapachula and Acapulco in the states of Chiapas and Guerrero, respectively.

Table 1. States and cities of collection sites, geographical coordinates and site's abbreviations used in this study.

\begin{tabular}{|c|c|c|c|c|c|}
\hline State & City & Site & Latitude & Longitude & Abbreviation \\
\hline \multicolumn{6}{|c|}{ Guerrero } \\
\hline & Acapulco & Zapata & 16.9049222 & -99.8410944 & Acp \\
\hline \multicolumn{6}{|c|}{ Chiapas } \\
\hline & Tapachula & Col.5 de Febrero & 14.9204944 & -92.2593472 & Tap \\
\hline \multicolumn{6}{|c|}{ Yucatan } \\
\hline & Merida & Fco. Montejo & 21.0307194 & -89.6463639 & Mer1 \\
\hline & & Plan Ayala & 21.0135833 & -89.6222222 & Mer2 \\
\hline & & U.H. Morelos & 20.9420139 & -89.5981556 & Mer3 \\
\hline & Conkal & Center & 21.0747917 & -89.5199056 & Co \\
\hline & Dzitya & Center & 21.0623278 & -89.6746694 & Dz \\
\hline & Acanceh & Center & 20.8126083 & -89.4505611 & Ac \\
\hline
\end{tabular}

\section{Establishment and maintenance of field populations}

$\mathrm{F}_{1}$ eggs were sent to Colorado State University. Egg papers were placed into a water container with $2 \mathrm{~L}$ of tap water to promote development and hatching. Larvae were fed $2 \mathrm{~mL}$ of $10 \%(\mathrm{w} / \mathrm{v})$ liver powder every other day. We transferred pupae to plastic cages for adult emergence. Larvae and mosquitoes were maintained in an incubator at $27-28^{\circ} \mathrm{C}, 70-80 \%$ humidity, and a photoperiod of $12 \mathrm{~h}$ light: $12 \mathrm{~h}$ dark. Adults were fed with raisins and allowed access to tap water. Females were offered citrated sheep blood on artificial membrane feeders, every four days, to obtain eggs. Females laid their eggs on moistened filter paper. The eggs were allowed to mature for $48 \mathrm{hr}$ before they were partially dried at room temperature 
and stored in sealed plastic bags. Each collection was split at the $\mathrm{F}_{1}$ larval stage into three groups of 50 to act as three biological replicates. In each subsequent generation $\sim 25$ adult $q$ and $25 \widehat{\diamond}$ were used to maintain each of the three replicates.

\section{Genotyping V1,016I and F1,534C}

DNA was extracted, at each generation $\left(\mathrm{F}_{1}-\mathrm{F}_{8}\right)$, from individual mosquitoes by the salt extraction method [6] and suspended in $180 \mu \mathrm{L}$ of TE buffer. To identify allelic variation we used the allele-specific polymerase chain reaction (asPCR), followed by generation of a melting curve (CFX-96 BioRad), to identify genotypes [7-9]. In each of the eight generations, we analyzed three replicates of 50 adult mosquitoes $(\sim 25 q$ and $25 ð)$ for each of the eight collection sites. Sample sizes were kept intentionally large to minimize founder's effects and genetic drift.

\section{Allele frequencies and linkage disequilibrium analysis.}

We estimated allele frequencies in each of the eight generations from the genotypic frequencies ((resistant allele frequency $=((2 *$ resistant homozygote $)+$ heterozygote $)) /(2 *$ sample size $))$. Allele frequencies were compared among replicates using a $2 \times 3$ contingency $\chi^{2}$ test. WINBugs 2.0 [10] with $10^{6}$ iterations was used to calculate the 95\% high-density intervals (HDI 95\%) around allele and genotype frequencies. We used ggplot2 in R-3.5.1 to graph the data. In addition, we used LINKDIS [11] and $\chi^{2}$ tests to calculate the pairwise linkage disequilibrium coefficients $\left(\mathrm{R}_{\mathrm{ij}}\right)$ between alleles at loci 1,016 and 1,534 [12] where:

$$
\mathrm{R}_{\mathrm{ij}}=\Delta_{\mathrm{ij}} / \sqrt{((p i(1-p i)+C i)(p j(1-p j)+C j))}
$$

where $\mathrm{Ci}=\operatorname{Hobs}(\mathrm{i})-\mathrm{p}_{\mathrm{i}}{ }^{2}$ and $\operatorname{Hobs}(\mathrm{i})$ is the observed frequency of $\mathrm{i}$ homozygotes and

$$
\Delta_{\mathrm{ij}}=(\mathrm{N} /(\mathrm{N}-1))\left(\left(\mathrm{T}_{\mathrm{ij}} / \mathrm{N}\right)-2 \mathrm{p}_{\mathrm{i}} \mathrm{p}_{\mathrm{j}}\right)
$$

$\mathrm{N}$ is the sample size and $\mathrm{p}_{\mathrm{i}}$ and $\mathrm{p}_{\mathrm{j}}$ are the frequencies of alleles at locus $\mathrm{i}=1,016$ and locus $\mathrm{j}=1,534$, respectively. $T_{i j}$ is the number of times that allele $i$ and allele $j$ occur in the same individual. A $\chi^{2}$ test was performed to determine if significant disequilibrium exists among all alleles at 1,016 and 1,534. The statistic was calculated and summed over all two-allele- interactions 


$$
\chi_{[1 \text { d.f. }]}^{2}=\mathrm{N} \sum_{i} \sum_{j}\left(\Delta_{\mathrm{ij}}^{2} / \mathrm{p}_{\mathrm{i}} \mathrm{p}_{\mathrm{j}}\right)
$$

\section{Resistance Ratio}

$\mathrm{LC}_{50}$ was calculated using New Orleans as a susceptible reference strain in all Resistance Ratio (RR) calculations. We calculated $\mathrm{LC}_{50}$ for New Orleans and for the 8 sites in generations $\mathrm{F}_{3}, \mathrm{~F}_{5}$ and $\mathrm{F}_{8}$ to evaluate changes in resistance in the eight collections. Values were calculated for permethrin and deltamethrin. We used PROC CORR in SAS 9.4 to calculate Pearson's correlation coefficient and to test for significance. This was done to examine the relationship between RR and the frequencies of I1,016 and $\mathrm{C} 1,534$ alleles. Analyses were performed in generations $\mathrm{F}_{3}, \mathrm{~F}_{6}$, and $\mathrm{F}_{8}$ and then for all three generations combined.

\section{Results}

\section{Frequency of I1,016 declines in the absence of pyrethroids.}

We determined the V1,016I genotype of 9,563 mosquitoes from eight locations in southern Mexico (Table 1) over eight generations $\left(\mathrm{F}_{1}-\mathrm{F}_{8}\right)$. The V1,016I genotype counts and allele frequencies appear in Appendices 1 and 2, respectively. Figure 1A plots I1,016 frequencies in all three replicates in the eight different collections over eight generations. In general, I1,016 allele frequencies were statistically uniform among the three replicates with five exceptions as indicated with an asterisk $\left(\mathrm{AcF}_{2}, \mathrm{CoF}_{2}, \mathrm{CoF}_{8}, \mathrm{DzF}_{2}\right.$,

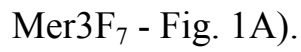

Figure 1B plots the mean I1,016 frequencies among all three replicates and the $95 \%$ high-density intervals (HDI 95\%). Pearson correlation coefficient between I1,016 frequencies and generation number and the associated significance appear at the bottom of each graph. The correlation between I1,016 frequency and generation number was negative and significant in four out of the eight collection sites from generation $\mathrm{F}_{1}$ to $\mathrm{F}_{8}$ (Ac, Acp, Co, and Mer2) (Fig. 1B). This correlation was not significant in sites Dz, Mer1 and Tap. I1,016 frequency in Co, Mer3 and Tap declined initially and then surprisingly 
increased in the last 3-4 generations. In general, I1,016 declined in frequency over eight generations, however, the rate and pattern of decline varied greatly among collections.

Fig 1. A) Plots of the frequencies of $\mathbf{I , 0 1 6}$ in all three replicates in the eight different collections over eight generations. Asterisk below the three replicates at a particular generation indicate when allele frequencies were statistically different among the three replicates.

B) Plots of the mean I1,016 frequencies among all three replicates and the $95 \%$ high-density intervals (HDI 95\%). Pearson correlation coefficient between I1,016 frequencies and generation number and the associated significance appear at the bottom of each graph.

V1,016I genotype frequencies $\left(\mathrm{VV}_{1,016}, \mathrm{VI}_{1,016}, \mathrm{II}_{1,016}\right)$ over eight generations are plotted in Fig. 2 in each of the 8 collections. Pearson correlations coefficients and significance between genotype frequencies and generation number appear in Table 2. The correlation between $\mathrm{VV}_{1,016}$ frequency and generation number were all positive and four were significant.

Fig 2. Frequencies of V1,016I genotype (VV1,016, VI1,016, II1,016) over eight generations. Six of the correlation coefficients for $\mathrm{VI}_{1,016}$ were positive and one was significant. All of the $\mathrm{II}_{1,016}$ were negative and six were significant.

Across all collections, there was a positive correlation $(r=0.4497, p=0.0002$ - Table 2$)$ between $\mathrm{VV}_{1,016}$ frequencies and generation number. In contrast, the frequencies of $\mathrm{VI}_{1,016}$ did not change over the eight generations $(r=0.1462, \mathrm{P}=0.2489$ - Table 2$)$ and were only significantly positive in Acp. $\mathrm{II}_{1,016}$ genotypic frequencies decreased significantly over generations $(r=-0.5452, \mathrm{P}<0.0001)$. In general there was an overall decline in the frequency of I1,016 and an overall increase in V1,016 over eight generations. 
Table 2. Pearson's correlation coefficient among collection sites V1,016I genotypic frequency and generations without exposure of pyrethroids.

\begin{tabular}{|c|c|c|c|c|c|c|}
\hline \multirow[b]{2}{*}{ Site } & \multicolumn{2}{|c|}{$\mathrm{VV}_{1,016}$} & \multicolumn{2}{|c|}{$\mathrm{VI}_{1,016}$} & \multicolumn{2}{|c|}{$\|_{1,016}$} \\
\hline & Pearson $r$ & $P$ value & Pearson $r$ & $P$ value & Pearson $r$ & $P$ value \\
\hline Ac & 0.9301 & 0.0008 & 0.6406 & 0.0870 & -0.9418 & 0.0005 \\
\hline Acp & 0.8156 & 0.0136 & 0.7455 & 0.0338 & -0.9619 & 0.0001 \\
\hline Co & 0.6499 & 0.0811 & -0.4268 & 0.2916 & -0.7886 & 0.0200 \\
\hline Dz & 0.2371 & 0.5718 & 0.1178 & 0.7811 & -0.4836 & 0.2247 \\
\hline Mer1 & 0.5472 & 0.1604 & 0.0404 & 0.9244 & -0.8005 & 0.0170 \\
\hline Mer2 & 0.8062 & 0.0156 & 0.3131 & 0.4502 & -0.8753 & 0.0044 \\
\hline Mer3 & 0.5830 & 0.1293 & 0.7033 & 0.0516 & -0.7085 & 0.0492 \\
\hline Tap & 0.7559 & 0.0300 & -0.1720 & 0.6838 & -0.3615 & 0.3789 \\
\hline Across all & 0.4497 & 0.0002 & 0.1462 & 0.2489 & -0.5452 & $<0.0001$ \\
\hline
\end{tabular}

Frequency of $\mathrm{C} 1,534$ declines in the absence of pyrethroids.

We genotyped F1,534C in each of the same mosquitoes for which V1,016I genotype frequencies had been determined. Appendix 1 lists the F1,534C genotypes counts, and Appendix 2 shows allele frequencies and the 95\% HDI. Figure 3A shows C1,534 frequencies in the eight collection sites over eight generations. C1,534 frequencies differed among replicates in 15 of the 64 comparisons.

Fig 3. A) Plots of the frequencies of $\mathbf{C 1 , 5 3 4}$ in all three replicates in the eight different collections over eight generations. Asterisk below the three replicates at a particular generation indicate when allele frequencies were statistically different among the three replicates.

B) Plots of the mean C1,534 frequencies among all three replicates and the $95 \%$ high-density intervals (HDI 95\%). Pearson correlation coefficient between $\mathrm{C} 1,534$ frequencies and generation number and the associated significance appear at the bottom of each graph.

The correlation coefficients between $\mathrm{C} 1,534$ frequencies and generation number and their significance are at the base of each of the graphs in Figure 3B. The correlation coefficients between $\mathrm{C} 1,534$ frequency and generation number was negative and significant in four out of the eight collection sites from generation $\mathrm{F}_{1}$ to $\mathrm{F}_{8}$ (Acp, Co, Mer1, and Mer2) (Fig. 3B). The correlation was not significant in sites Ac, Dz, Mer3 and Tap. In general, C1,534 declined in frequency over eight generations, however, the rate and pattern of decline varied greatly among collections. 
Fig 4. Frequencies of $\mathrm{F} 1,534$ genotype $\left(\mathrm{FF}_{1,534}, \mathrm{FC}_{1,534}, \mathrm{CC}_{1,534}\right)$ over eight generations.

Correlations between genotype frequencies and generation are provided in Table 3.

Table 3. Pearson's correlation coefficient among collection sites F1,534C genotypic frequency and generations without exposure of pyrethroids.

\begin{tabular}{|c|c|c|c|c|c|c|}
\hline \multirow[b]{2}{*}{ Site } & \multicolumn{2}{|c|}{$\mathrm{FF}_{1,534}$} & \multicolumn{2}{|c|}{$\mathrm{FC}_{1,534}$} & \multicolumn{2}{|c|}{$\mathrm{CC}_{1,534}$} \\
\hline & Pearson $r$ & $P$ value & Pearson $r$ & $P$ value & Pearson $r$ & $P$ value \\
\hline Ac & -0.363 & 0.3759 & 0.6537 & 0.0788 & -0.5460 & 0.1615 \\
\hline Acp & 0.6952 & 0.0556 & 0.9711 & $<0.0001$ & -0.9110 & 0.0016 \\
\hline Co & 0.8953 & 0.0026 & 0.0528 & 0.9012 & -0.8996 & 0.0023 \\
\hline Dz & 0.3782 & 0.3555 & 0.3733 & 0.3624 & -0.5175 & 0.189 \\
\hline Mer1 & 0.2729 & 0.5131 & -0.8254 & 0.0116 & 0.7953 & 0.0183 \\
\hline Mer2 & 0.7997 & 0.0172 & 0.8748 & 0.0045 & -0.9131 & 0.0015 \\
\hline Mer3 & -0.3423 & 0.4066 & -0.0164 & 0.9692 & 0.1308 & 0.7575 \\
\hline Tap & -0.5774 & 0.1340 & -0.2701 & 0.5177 & 0.3309 & 0.4233 \\
\hline Across all & 0.3399 & 0.006 & 0.2078 & 0.0994 & -0.3024 & 0.0152 \\
\hline
\end{tabular}

Five correlation coefficients for $\mathrm{FF}_{1,534}$ were positive and two were significant, five of the correlation

coefficients for $\mathrm{FC}_{1,534}$ were positive and three were significant and five of the $\mathrm{CC}_{1,534}$ were negative and four were significant. Across all collections sites, there was a positive correlation $(r=0.3399, p=0.006)$ between $\mathrm{FF}_{1,534}$ frequencies and generation number. The frequencies of $\mathrm{FC}_{1,016}$ did not change significantly over generations $(r=0.2078, \mathrm{P}=0.0994)$ and as expected for a genotype that confers a lower fitness, $\mathrm{CC}_{1,534}$ genotypic frequencies decreased significantly over generations $(r=-0.3024, \mathrm{P}=0.0152)$.

\section{Linkage disequilibrium}

We performed pairwise linkage disequilibrium analyses between alleles in V1,016I and F1,534C. Table 4 lists the linkage disequilibrium coefficients $\mathrm{R}_{\mathrm{ij}}, \chi^{2}$ and the probability value obtained between pairwise loci. Rij is distributed from -1.00 (mutations occur on opposite chromosomes - trans) to 0.00 (mutations occur independently), to 1.00 (both mutations cis on the same chromosome) and therefore provides a standardized measure of disequilibrium.

Alleles segregated in 57 out of 64 collections (Table 4). Forty seven of the 57 collections exhibited significant linkage disequilibrium, with $\mathrm{R}_{\mathrm{ij}}$ values ranging between 0.15-0.85 among collections. In general and in agreement with earlier studies $[13,14]$ alleles in V1,016I and F1,534C were in linkage disequilibrium. 
Fig. 5 Plots the frequency of the four potential dilocus haplotypes over eight generations.

Table 5 displays the correlation and the significance between haplotype frequencies and generation.

Table 5. Pearson's correlation and $p$ value for four haplotypes at di-locus V1016I/F1534C.

\begin{tabular}{|c|c|c|c|c|c|c|c|c|}
\hline \multirow[b]{2}{*}{ Site } & \multicolumn{2}{|c|}{$V_{1,016} / F_{1,534}$} & \multicolumn{2}{|c|}{$V_{1,016} / C_{1,534}$} & \multicolumn{2}{|c|}{$I_{1,016} / F_{1,534}$} & \multicolumn{2}{|c|}{$I_{1,016} / C_{1,534}$} \\
\hline & Pearson $r$ & $P$ value & Pearson $r$ & $P$ value & Pearson $r$ & $P$ value & Pearson $r$ & $P$ value \\
\hline Ac & 0.282 & 0.500 & 0.868 & 0.005 & 0.512 & 0.195 & -0.978 & $<0.0001$ \\
\hline Acp & 0.842 & 0.009 & -0.356 & 0.386 & 0.432 & 0.285 & -0.937 & 0.001 \\
\hline Co & 0.754 & 0.031 & -0.566 & 0.143 & 0.401 & 0.325 & -0.907 & 0.002 \\
\hline Dz & 0.429 & 0.289 & -0.251 & 0.549 & 0.130 & 0.759 & -0.388 & 0.342 \\
\hline Mer1 & -0.268 & 0.521 & 0.873 & 0.005 & -0.672 & 0.068 & -0.618 & 0.103 \\
\hline Mer2 & 0.882 & 0.004 & -0.633 & 0.092 & -0.339 & 0.412 & -0.770 & 0.026 \\
\hline Mer3 & -0.340 & 0.410 & 0.677 & 0.065 & -0.031 & 0.942 & -0.688 & 0.059 \\
\hline Tap & -0.480 & 0.229 & 0.624 & 0.099 & -0.051 & 0.904 & -0.560 & 0.149 \\
\hline Overall & 0.323 & 0.009 & 0.140 & 0.271 & 0.091 & 0.474 & -0.516 & $<0.0001$ \\
\hline
\end{tabular}

The frequency of the susceptible $\mathrm{V}_{1,016} / \mathrm{F}_{1,534}$ haplotype increased over generations $(r=0.323, p=$

0.009). The frequency of the $\mathrm{V}_{1,016} / \mathrm{C}_{1,534}$ haplotype remained relatively constant $(r=0.140, p=0.271)$.

The frequency of the resistant $\mathrm{I}_{1,016} / \mathrm{C}_{1,534}$ haplotype decreased over time $(r=-0.516, p<0.0001)$ across all collection sites. The frequencies of the $\mathrm{I}_{1,016} / \mathrm{F}_{1,534}$ haplotypes were consistently low $(r=0.091, p=0.474)$ across generations. This same trend has been noted in two previous studies [5] [15] and suggested that low fitness may occur in any mosquito in which I1,016 co-occurs with F1,534.

Temporal analysis of di-locus genotypes.

Figure 6 shows the frequency of nine di-locus genotype combinations ( 3 genotypes at 2 loci) and Table 6 lists the correlation between frequencies of each di-locus genotype and the generation number without pyrethroid exposure. We estimated the frequencies of the nine genotype combinations in 9,563 mosquitoes. However, the di-locus coefficient among the nine graphs was only significant for the wild type susceptible $\mathrm{VV}_{1016} / \mathrm{FF}_{1534}$ and the dual resistant $\mathrm{II}_{1016} / \mathrm{CC}_{1534}$. The correlation between generation number and $\mathrm{VV}_{1016} / \mathrm{FF}_{1534}$ was positive $(\mathrm{r}=0.3376, \mathrm{P}=0.0064)$ indicating an increase while the 
correlation of $\mathrm{II}_{1016} / \mathrm{CC}_{1534}$. Was negative $(\mathrm{r}=-0.5465, \mathrm{P}<0.0001)$ indicating a decline. The low frequencies of $\mathrm{VI}_{1,016} / \mathrm{FF}_{1,534}, \mathrm{II}_{1,016} / \mathrm{FF}_{1,534}$, and $\mathrm{II}_{1,016} / \mathrm{FC}_{1,534}$ in Figs 6 B,C,F are consistent with the

Fig 6. Frequency of the nine dilocus haplotypes over eight generations.

hypothesis that low fitness may occur in any mosquito in which I1,016 co-occurs with F1,534. However Figure $6 \mathrm{E}$ identifies an exception to this trend. A substantial proportion of the double heterozygote $\mathrm{VI}_{1016} / \mathrm{FC}_{1534}$ survive to generation $\mathrm{F}_{8}$.

\section{Association between resistant allele frequencies and the Resistance Ratio.}

Pearson correlation coefficients and their significance were calculated between the frequencies of I1,016 or $\mathrm{C} 1,534$ alleles and resistance ratios for permethrin and deltamethrin as determined by bioassay for all eight collections. This was done separately for generations $\mathrm{F}_{3}, \mathrm{~F}_{6}$, and $\mathrm{F}_{8}$. Table 7 indicates that all correlations were positive in $\mathrm{F}_{3}$ and $\mathrm{F}_{8}$ but none were significant. All correlations were positive in $\mathrm{F}_{6}$ and

Table 7. Pearson correlation coefficients and their significance were calculated between the frequencies of $\mathrm{I1,016}$ or $\mathrm{C} 1,534$ alleles and resistance ratios as determined by bioassay for all eight collections. Significant values appear in bold.

Locus

Generation

F3 (n=9)

F6 ( $\mathrm{n}=9)$

$\mathrm{F} 8(\mathrm{n}=9)$

All $(n=27)$

Locus

Generation

F3 (n=9)

F6 $(n=9)$
I1,1016

Correlation of allele frequency with Permethrin

RR

0.606

0.715

0.338

0.588

C1534

Correlation of allele frequency with Permethrin

RR

0.711

0.822
Prob*

0.1489

0.0303

0.3732

0.002
Correlation of allele frequency with Prob Deltamethrin RR

0.625

0.184

0.709

0.607

0.469

0.083

0.021
Correlation of allele frequency with

Prob

0.073

0.007
Deltamethrin RR

0.66

0.593
Prob

0.154

0.093 


$\begin{array}{ccccc}\mathrm{F} 8(\mathrm{n}=9) & 0.115 & 0.768 & 0.282 & 0.462 \\ \mathrm{All}(\mathrm{n}=27) & \mathbf{0 . 7 0 7} & <.0001 & 0.367 & 0.078\end{array}$

three were significant (in bold). When combining results from all three generations together, all correlations were positive and three were significant (in bold). Thus in general there was a weak but consistently positive correlation between the frequency of $\mathrm{I} 1,016$ or $\mathrm{C} 1,534$ alleles and resistance ratios as determined by bioassay.

This was noted in an earlier study in which I1,016 showed significant protection against permethrin, and deltamethrin whereas F1534C showed protection against permethrin but not against deltamethrin [16, 17]. The expression of $\mathrm{C} 1,534$ in Xenopus oocytes and exposure to both pyrethroids demonstrated that the resistant amino acid substitution in $\mathrm{C} 1,534$ is sensitive to permethrin but not for deltamethrin, which is consistent with our results.

\section{Discussion}

In this study we established eight colonies of Ae. aegypti from the field and maintained them in a pyrethroid free environment in the laboratory over eight generations. We demonstrated that in general the frequency of the Ae. aegypti pyrethroid resistance alleles I1,016 and C1,534 decline when released from pyrethroid pressure in the laboratory (Figs. 1 and 3, Tables 3 and 5). However, the pattern of decline appeared to be strain dependent with some having a steady rate of decline (Ac, Acp, Mer2 in Fig. 1, Acp and Mer2 in Fig. 3), some showing a shallow decline (e.g. Mer1, Tap in Fig 1; Acp, Mer2and Co in Fig. 3) and others displaying no net change (Fig.1 Dz; Fig 3.Ac, Mer3, and Tap).

A more surprising result was that in Co and Mer3, the frequencies of I1,016 actually increased following a precipitous drop. Likewise the frequencies of C1,534 in Mer1, Mer2, and Co, increased after a drop. This might occur if there are deleterious or lethal recessive mutations linked to the susceptible allele that became homozygous through continuous inbreeding. However this theory fails to explain why in Co I1,016 increased simultaneously in all three replicates. 
We do not propose nor do we have any idea as to whether the selection pressure in indoor cage studies is the same as or is even correlated with outdoor selection pressure. This study only indicates that the loss of pyrethroid resistance is unlikely to follow a smooth linear or exponential decline for any one of a number of reasons. Nor should we expect the decline in resistance to be consistent among collections. Epistatic interactions between alleles may cause non-linear trends in allele frequencies. Much depends on the genetic background of each population; some populations could take much longer to lose resistance while others may be much faster.

There is a variety of possible causes for this variance in gene frequency trajectories among collections but it is unlikely that the heterogeneity arose from small sample sizes. We analyzed three replicates of 50 adult mosquitoes for each of the eight collection sites. Furthermore, 50 adults were used to generate the next generation of eggs for each replicate. The $95 \%$ HDI remained narrow in all graphs in Fig1B and Fig. 3B.

Initial conditions may affect the shape of the curve. For example, a curve that begins with initial frequencies close to 1 (Fig. 3A, Mer3 and Tap) would begin to decline much later than a curve that begins at 0.6 (Fig 1A, Mer1, Mer2). Metabolic resistance may account for much of this heterogeneity. A QTL mapping study [9] reported that $58.6 \%$ of the variation in knockdown could be accounted for by I1,016 but that a number of different QTL located throughout the genome accounted for the remainder of the variation. Saavedra-Rodriguez et al used the 'Aedes Detox' microarray [18] and showed an inverse relationship between I1,016 frequencies and the numbers of differentially transcribed metabolic genes[19].

Table 5 displays the correlation and the significance between haplotype frequencies and generation. The low frequencies of $\mathrm{VI}_{1,016} / \mathrm{FF}_{1,534}, \mathrm{II}_{1,016} / \mathrm{FF}_{1,534}$, and $\mathrm{II}_{1,016} / \mathrm{FC}_{1,534}$ noted in this (Fig 6 B,C,F) and two previous studies [5], [15] suggests that low fitness may occur in any mosquito in which I1,016 co-occurs with F1,534. This and the fact that C1,534 historically appears before I1,016 argues that evolution of the mutations were sequential. If I1,016 had appeared first it would have co-occured with F1,534 and would have been eliminated. 
Deltamethrin RR appears to be correlated with I1,016 allele frequency but not with C1,534 allele frequencies, while permethrin RR correlates with both allele frequencies (Table 7). We speculated that there are other resistant mechanisms that could drive resistance to deltamethrin, such as detoxifying enzymes or other mutations in $V G S C$ that have not been identified. However, permethrin resistance appears to be driven heavily by both resistance alleles.

The I1,016, amino acid substitution has been found in many resistant populations in the Americas [20-25] and it has been shown that it is in linkage disequilibrium with C1,534 [12]. Recent work has shown that I1,016 is also in very tight disequilibrium with V410L [16].

Interestingly, the I1,016 mutation was functionally expressed in Xenopus oocytes and did not show an alteration to the sodium channel sensitivity to both pyrethroids [16]. Our data indicate a positive correlation of I1,016 allele frequency with the RR of both pyrethroids. It is clear that more understanding is needed as to the role that I1,016 plays in resistance of Ae. aegypti. This includes the possibility that V410L [16] may be the residue to which the pyrethroid actually binds.

Since many field populations are resistant to pyrethroid and few insecticides are available in the market, negative fitness is beneficial for vector control, providing an opportunity where alternative insecticide could be used while losing pyrethroid resistance. Pyrethroids could then be "saved" to control susceptible populations during a disease outbreak. However it is important to remember that even if dominant wild type alleles (V1,016 and F1,534) increase in a population, recessive resistance alleles will be hidden and maintained. There is no way that the initial susceptibility of populations (before the introduction of a pyrethroid) will ever be recovered. Recent work in Sao Paulo State in Brazil showed that resistance alleles persist in natural populations for at least 11 years [1] .

\section{Acknowledegments}

This study was funded by the National Institutes of Health/National Institute of Allergy and Infectious Diseases International Collaborations in Infectious Disease Research Program (U01-AI-088647) and 
bioRxiv preprint doi: https://doi.org/10.1101/760710; this version posted September 6,2019 . The copyright holder for this preprint (which was not certified by peer review) is the author/funder, who has granted bioRxiv a license to display the preprint in perpetuity. It is made available under aCC-BY 4.0 International license.

"Insecticide Resistance Management to Preserve Pyrethroid Resistance in Aedes aegypti"

(1R01AI121211-01A1). Farah Vera-Maloof and Patricia Penilla were supported by the Fogarty Training

Grant "Training in Dengue Prevention and Control” (2D43TW001130-08). 


\section{References}

1. Macoris MD, Martins AJ, Andrighetti MTM, Lima JBP, Valle D. Pyrethroid resistance persists after ten years without usage against Aedes aegypti in governmental campaigns: Lessons from Sao Paulo State, Brazil. Plos Neglected Tropical Diseases. 2018;12(3). doi: ARTN $\mathrm{e} 0006390$

10.1371/journal.pntd.0006390. PubMed PMID: WOS:000431268900085.

2. Amelia-Yap ZH, Chen CD, Sofian-Azirun M, Low VL. Pyrethroid resistance in the dengue vector Aedes aegypti in Southeast Asia: present situation and prospects for management. Parasites \& Vectors. 2018;11. doi: ARTN 332

10.1186/s13071-018-2899-0. PubMed PMID: WOS:000434235600001.

3. Chang C, Huang XY, Chang PC, Wu HH, Dai SM. Inheritance and stability of sodium channel mutations associated with permethrin knockdown resistance in Aedes aegypti. Pesticide Biochemistry and Physiology. 2012;104(2):136-42. doi: 10.1016/j.pestbp.2012.06.003. PubMed PMID: WOS:000311068900010.

4. Brito LP, Linss JGB, Lima-Camara TN, Belinato TA, Peixoto AA, Lima JBP, et al. Assessing the Effects of Aedes aegypti kdr Mutations on Pyrethroid Resistance and Its Fitness Cost. Plos One. 2013;8(4). doi: ARTN e60878

10.1371/journal.pone.0060878. PubMed PMID: WOS:000317898000080.

5. Grossman MK, Uc-Puc V, Rodriguez J, Cutler DJ, Morran LT, Manrique-Saide P, et al. Restoration of pyrethroid susceptibility in a highly resistant Aedes aegypti population. Biology Letters. 2018;14(6). doi: ARTN 20180022

10.1098/rsbl.2018.0022. PubMed PMID: WOS:000437005700002. 
6. Black WC, DuTeau NM. RAPD-PCR and SSCP analysis for insect population genetic studies. The Molecular Biology of Insect Disease Vectors: A Methods Manual. Crampton J BC LC, editor, editor. New York: Chapman and Hall; 1997.

7. Yanola J, Somboon P, Walton C, Nachaiwieng W, Somwang P, Prapanthadara LA. Highthroughput assays for detection of the F1534C mutation in the voltage-gated sodium channel gene in permethrin-resistant Aedes aegypti and the distribution of this mutation throughout Thailand. Tropical Medicine \& International Health. 2011;16(4):501-9. doi: 10.1111/j.13653156.2011.02725.x. PubMed PMID: WOS:000288509500013.

8. Saavedra-Rodriguez K, Urdaneta-Marquez L, Rajatileka S, Moulton M, Flores AE, Fernandez-Salas I, et al. A mutation in the voltage-gated sodium channel gene associated with pyrethroid resistance in Latin American Aedes aegypti. Insect Molecular Biology. 2007;16(6):785-98. doi: DOI 10.1111/j.1365-2583.2007.00774.x. PubMed PMID: WOS:000251739500013.

9. Saavedra-Rodriguez K, Strode C, Suarez AF, Salas IF, Ranson H, Hemingway J, et al. Quantitative Trait Loci Mapping of Genome Regions Controlling Permethrin Resistance in the Mosquito Aedes aegypti. Genetics. 2008;180(2):1137-52. doi: 10.1534/genetics.108.087924. PubMed PMID: WOS:000260284400036.

10. Puza B. Inference via WinBUGS. Bayesian Methods for Statistical Analysis. 2015:365406. PubMed PMID: WOS:000379227300010.

11. Black WC, Krafsur ES. A FORTRAN program for the calculation and analysis of twolocus linkage disequilibrium coefficients. Theor Appl Genet. 1985;70:491-6.

12. Vera-Maloof FZ, Saavedra-Rodriguez K, Elizondo-Quiroga AE, Lozano-Fuentes S, Black WC. Coevolution of the Ile1,016 and Cys1,534 Mutations in the Voltage Gated Sodium 
Channel Gene of Aedes aegypti in Mexico. PLoS Negl Trop Dis. 2015;9(12):e0004263. Epub 2015/12/15. doi: 10.1371/journal.pntd.0004263. PubMed PMID: 26658798; PubMed Central PMCID: PMCPMC4684211.

13. Saavedra-Rodriguez K, Maloof FV, Campbell CL, Garcia-Rejon J, Lenhart A, Penilla P, et al. Parallel evolution of vgsc mutations at domains IS6, IIS6 and IIIS6 in pyrethroid resistant Aedes aegypti from Mexico. Scientific Reports. 2018;8(1):6747. doi: 10.1038/s41598-01825222-0.

14. Vera-Maloof FZ, Saavedra-Rodriguez K, Elizondo-Quiroga AE, Lozano-Fuentes S, Black Iv WC. Coevolution of the Ile1,016 and Cys1,534 Mutations in the Voltage Gated Sodium Channel Gene of Aedes aegypti in Mexico. PLoS Negl Trop Dis. 2015;9(12):e0004263. Epub 2015/12/15. doi: 10.1371/journal.pntd.0004263. PubMed PMID: 26658798; PubMed Central PMCID: PMCPMC4684211.

15. Saavedra-Rodriguez K, Maloof FV, Campbell CL, Garcia-Rejon J, Lenhart A, Penilla P, et al. Parallel evolution of vgsc mutations at domains IS6, IIS6 and IIIS6 in pyrethroid resistant Aedes aegypti from Mexico. Sci Rep-Uk. 2018;8. doi: ARTN 6747

10.1038/s41598-018-25222-0. PubMed PMID: WOS:000431107100006.

16. Du Y, Nomura Y, Satar G, Hu Z, Nauen R, He SY, et al. Molecular evidence for dual pyrethroid-receptor sites on a mosquito sodium channel. Proc Natl Acad Sci U S A. 2013;110(29):11785-90. Epub 2013/07/04. doi: 10.1073/pnas.1305118110. PubMed PMID: 23821746; PubMed Central PMCID: PMCPMC3718148.

17. Haddi K, Tome HVV, Du Y, Valbon WR, Nomura Y, Martins GF, et al. Detection of a new pyrethroid resistance mutation (V410L) in the sodium channel of Aedes aegypti: a potential 
challenge for mosquito control. Sci Rep. 2017;7:46549. Epub 2017/04/20. doi:

10.1038/srep46549. PubMed PMID: 28422157; PubMed Central PMCID: PMCPMC5396194.

18. Strode C, Karunarate PS, Black WC, Ranson H. The differential gene expression of detoxification enzymes implicated in insecticide resistance of aedes aegypti using a small scale microarray. Am J Trop Med Hyg. 2006;75(5):5-. PubMed PMID: WOS:000242343900018.

19. Saavedra-Rodriguez K, Suarez AF, Salas IF, Strode C, Ranson H, Hemingway J, et al. Transcription of detoxification genes after permethrin selection in the mosquito Aedes aegypti. Insect Molecular Biology. 2012;21(1):61-77. doi: 10.1111/j.1365-2583.2011.01113.x. PubMed PMID: WOS:000299101500007.

20. Garcia GP, Flores AE, Fernandez-Salas I, Saavedra-Rodriguez K, Reyes-Solis G, Lozano-Fuentes S, et al. Recent rapid rise of a permethrin knock down resistance allele in Aedes aegypti in Mexico. PLoS Negl Trop Dis. 2009;3(10):e531. Epub 2009/10/16. doi:

10.1371/journal.pntd.0000531. PubMed PMID: 19829709; PubMed Central PMCID: PMCPMC2759509.

21. Bona AC, Chitolina RF, Fermino ML, de Castro Poncio L, Weiss A, Lima JB, et al. Larval application of sodium channel homologous dsRNA restores pyrethroid insecticide susceptibility in a resistant adult mosquito population. Parasit Vectors. 2016;9(1):397. Epub 2016/07/16. doi: 10.1186/s13071-016-1634-y. PubMed PMID: 27416771; PubMed Central PMCID: PMCPMC4946210.

22. Chapadense FG, Fernandes EK, Lima JB, Martins AJ, Silva LC, Rocha WT, et al. Phenotypic and genotypic profile of pyrethroid resistance in populations of the mosquito Aedes aegypti from Goiania, Central West Brazil. Rev Soc Bras Med Trop. 2015;48(5):607-9. Epub 2015/11/01. doi: 10.1590/0037-8682-0046-2015. PubMed PMID: 26516973. 
23. Linss JG, Brito LP, Garcia GA, Araki AS, Bruno RV, Lima JB, et al. Distribution and dissemination of the Val1016Ile and Phe1534Cys Kdr mutations in Aedes aegypti Brazilian natural populations. Parasit Vectors. 2014;7:25. Epub 2014/01/17. doi: 10.1186/1756-3305-7-25. PubMed PMID: 24428880; PubMed Central PMCID: PMCPMC3912884.

24. Aponte HA, Penilla RP, Dzul-Manzanilla F, Che-Mendoza A, Lopez AD, Solis F, et al. The pyrethroid resistance status and mechanisms in Aedes aegypti from the Guerrero state, Mexico. Pesticide Biochemistry and Physiology. 2013;107(2):226-34. doi:

10.1016/j.pestbp.2013.07.005. PubMed PMID: WOS:000326208000009.

25. Cornel AJ, Holeman J, Nieman CC, Lee Y, Smith C, Amorino M, et al. Surveillance, insecticide resistance and control of an invasive Aedes aegypti (Diptera: Culicidae) population in California. F1000Res. 2016;5:194. Epub 2016/05/10. doi: 10.12688/f1000research.8107.3.

PubMed PMID: 27158450; PubMed Central PMCID: PMCPMC4857756. 
A
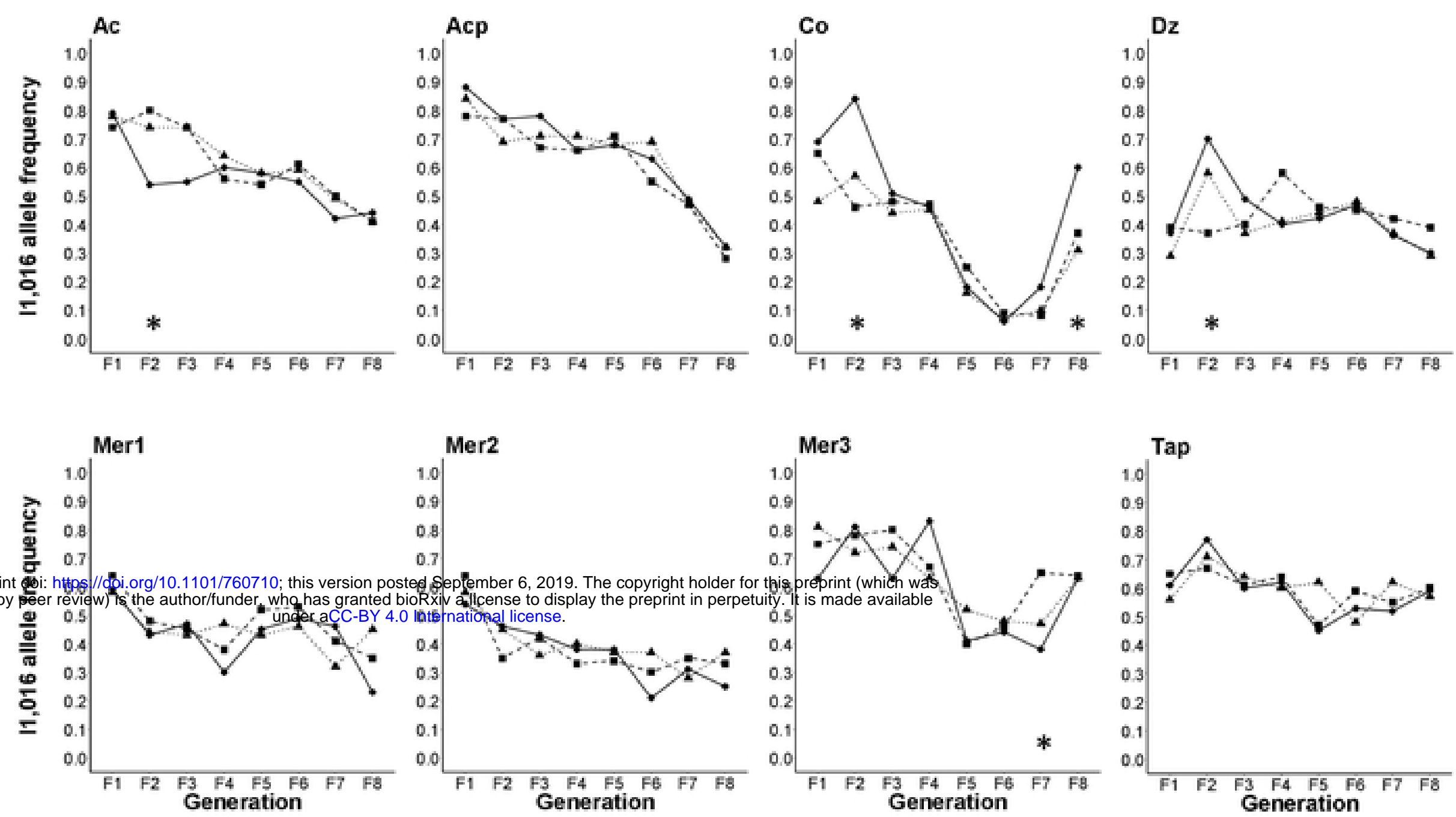

B
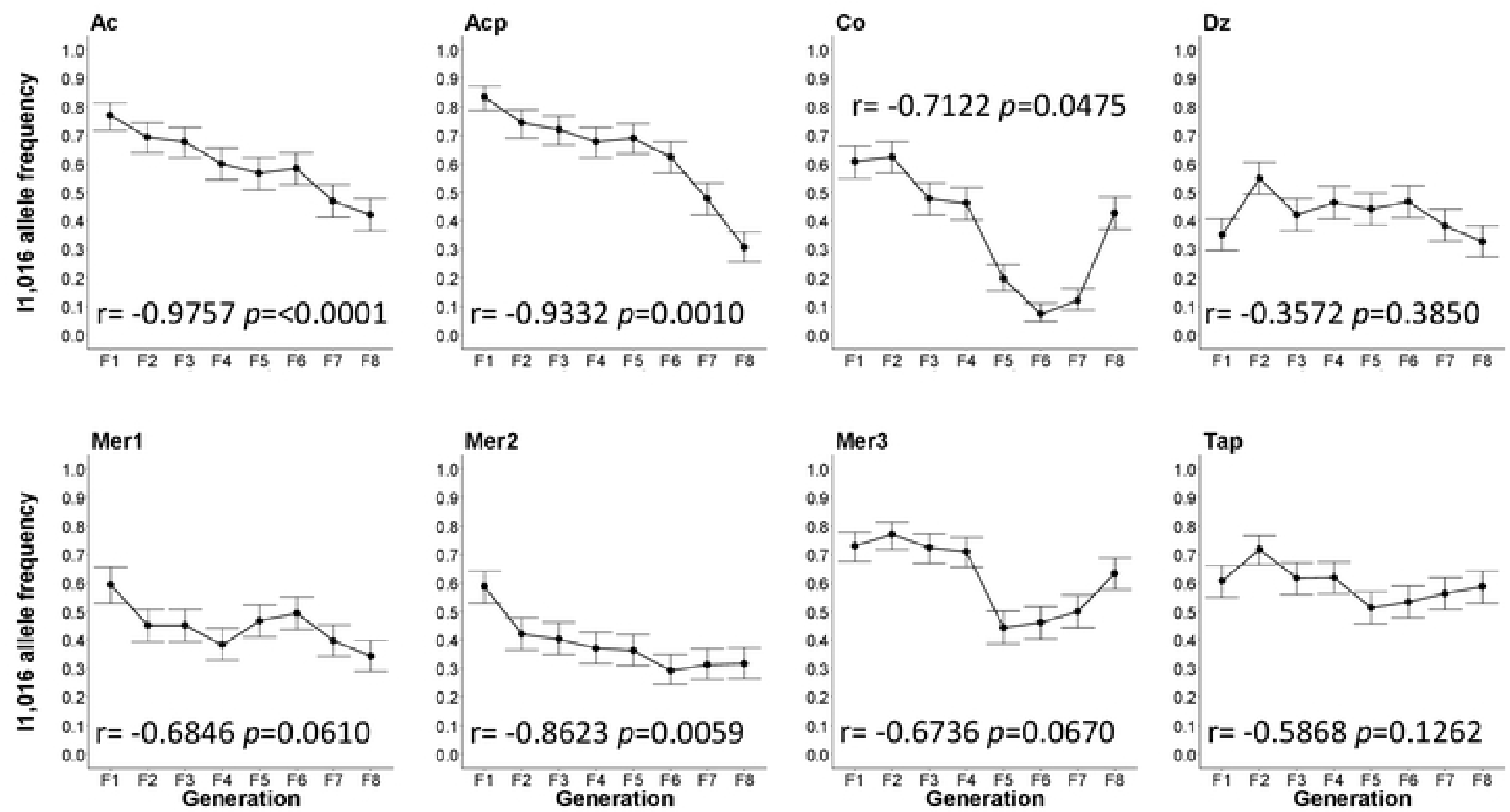

Fig1 

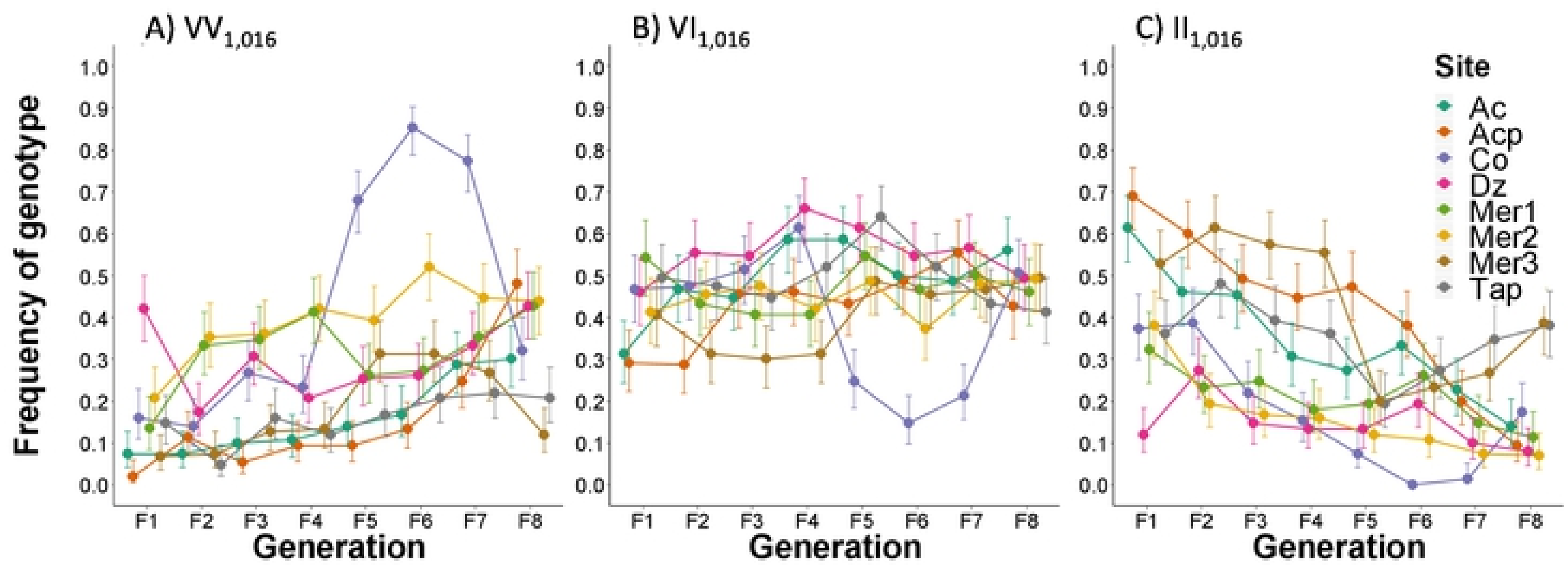

Fig2 
A
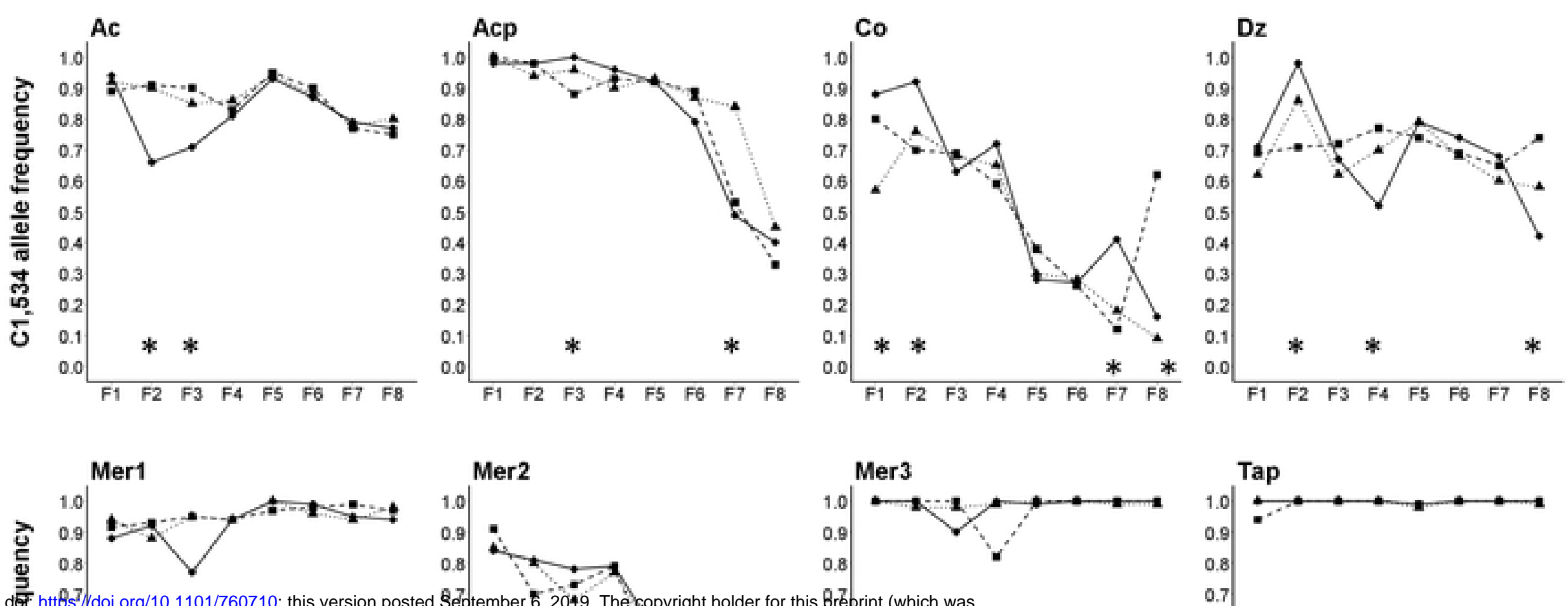

bioRxiv preprint d $d$ : httBs. / /doi. org/10.1101/760710 this version posted September 2049 . The copyright holder for this Breprint (which was

not certified by peger review) is the author/funder, who has gand
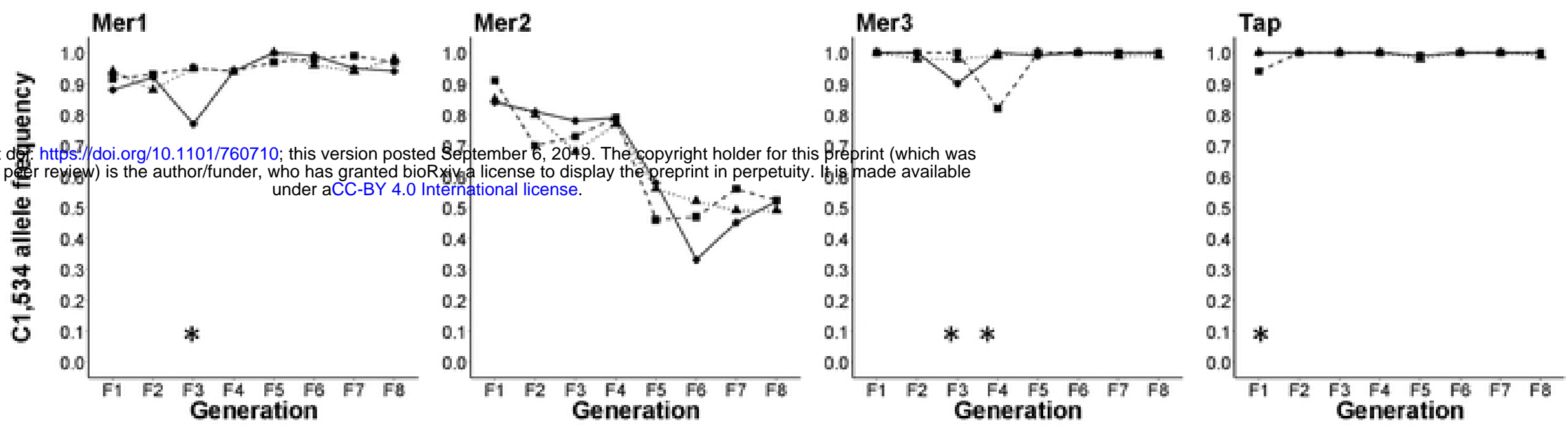

B
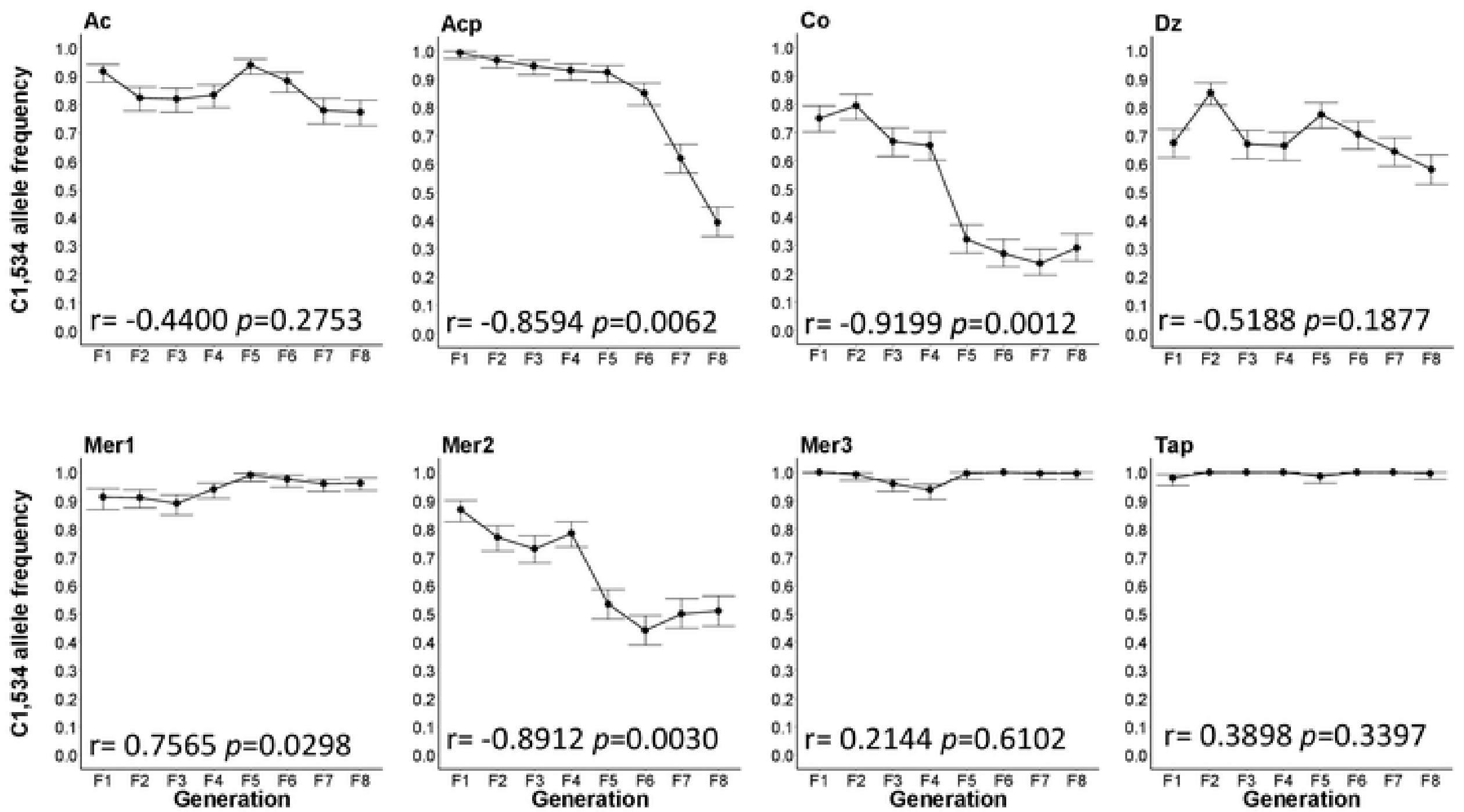

Fig3 

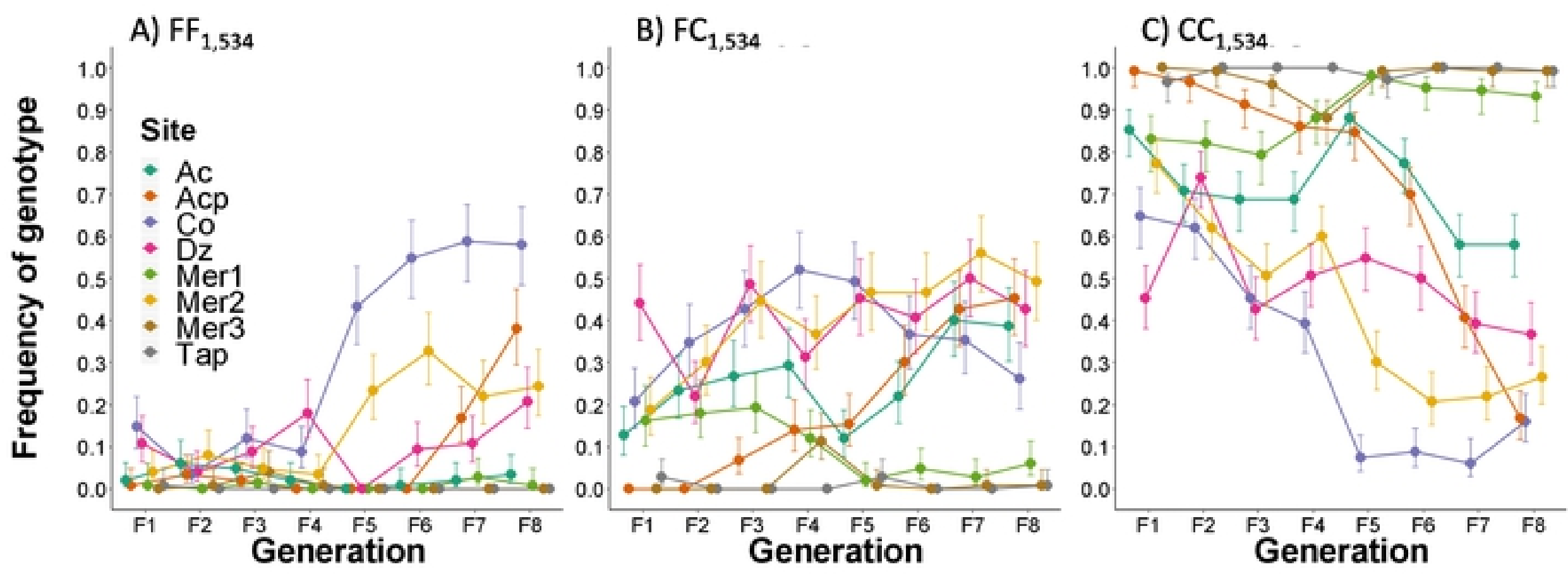

Fig4 

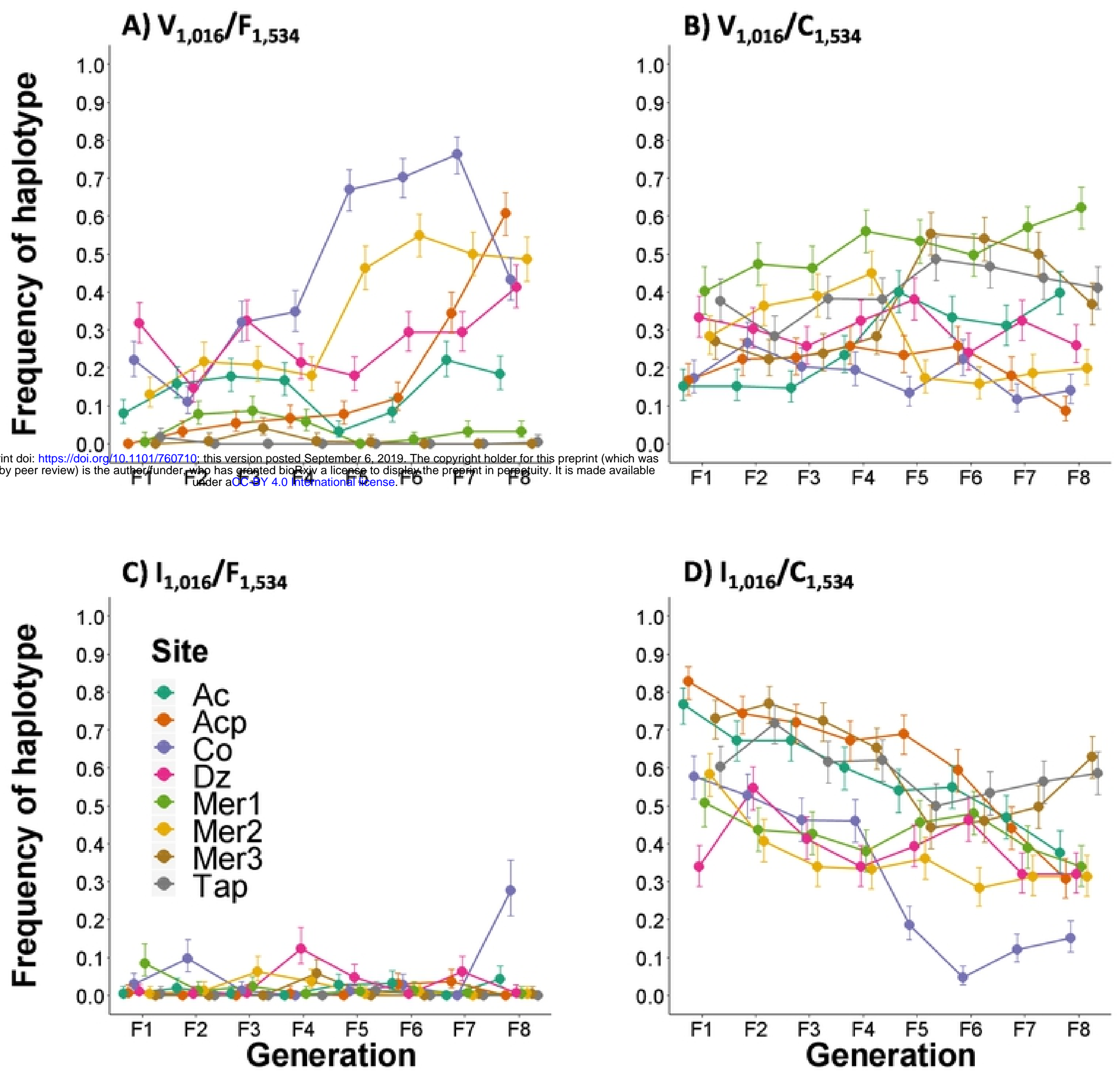

Fig5 

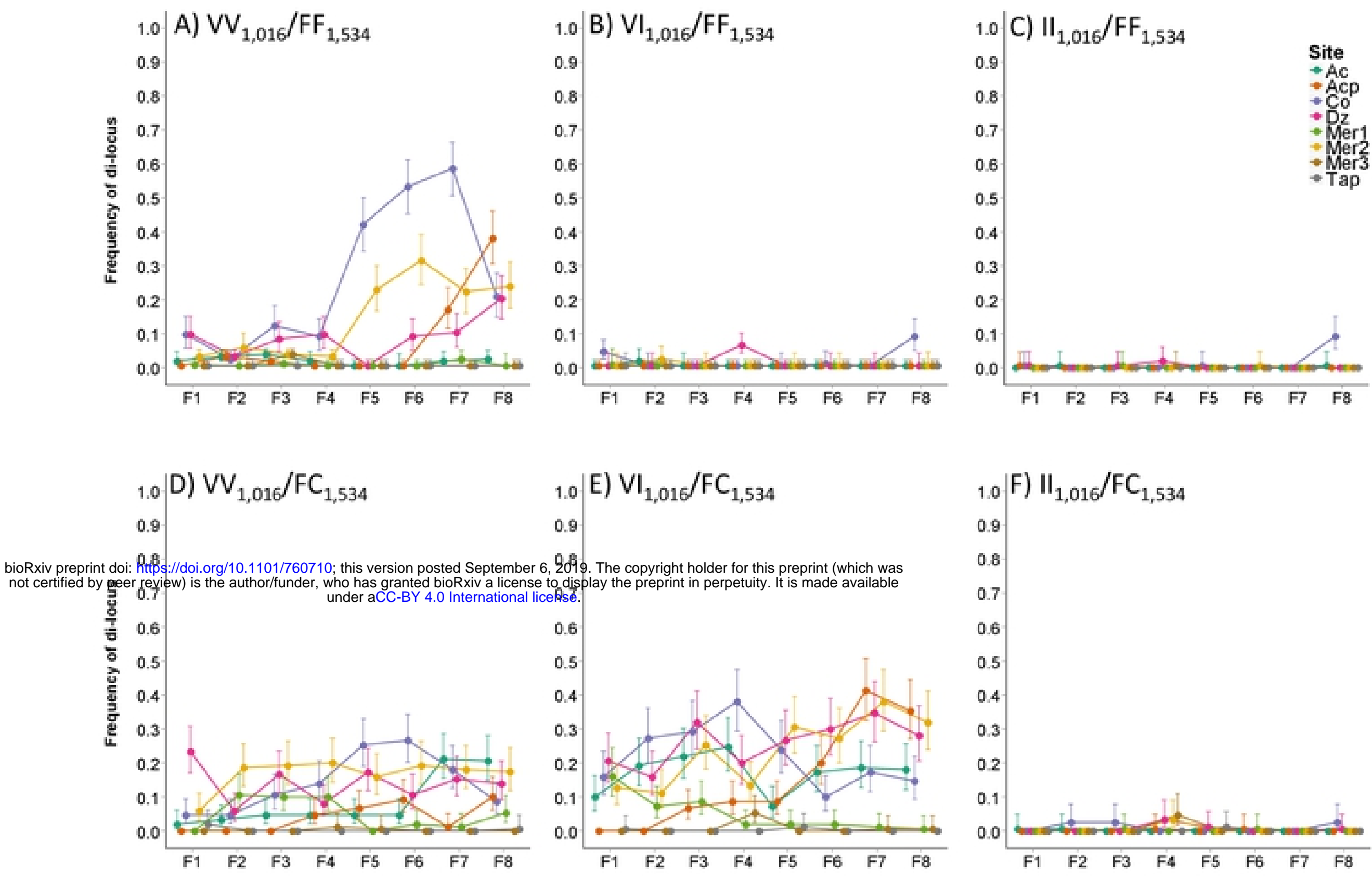

2019. The copyright holder for this preprint (which was
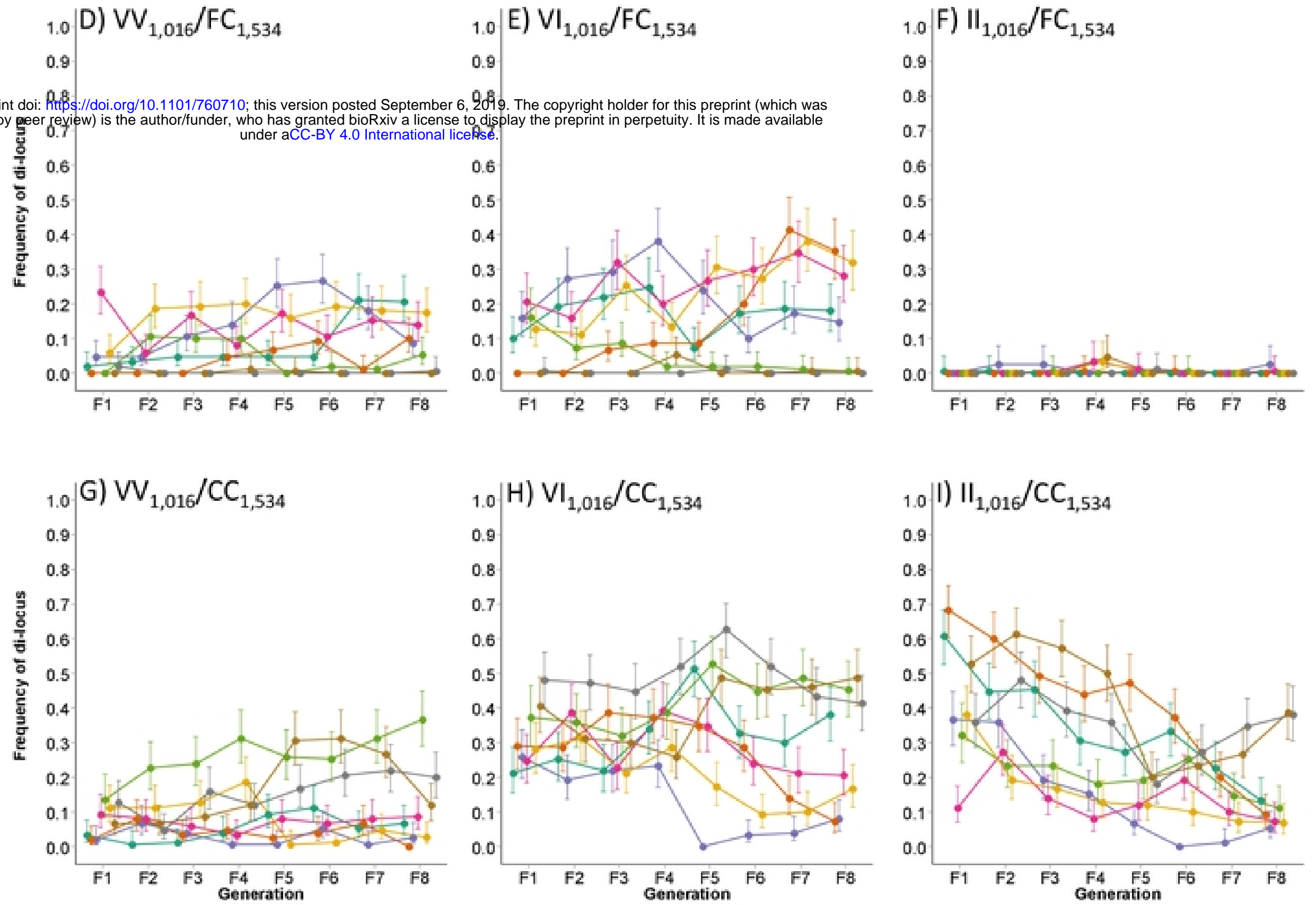